\title{
An Unusual Case of Isolated Cryptococcal Osteomyelitis of Mastoid: An Enigma!
}

\author{
Dharanya Srinivasan ${ }^{1}$ Subhashini Puducherry Ravichandran ${ }^{1} \quad$ Karthikeyan Ramasamy ${ }^{1}$ \\ Sivaraman Ganesan ${ }^{1}$ Arun Alexander ${ }^{1}$
}

${ }^{1}$ Department of ENT, Jawaharlal Institute of Postgraduate Medical Education and Research, Puducherry, India

\begin{abstract}
Address for correspondence Subhashini Puducherry Ravichandran, MS, DNB, Department of ENT, Jawaharlal Institute of Postgraduate Medical Education and Research, Puducherry 605006, India (e-mail: prsubhashini90@gmail.com).
\end{abstract}

Ann Otol Neurotol:2020;3:42-45

\begin{abstract}
Keywords

- cryptococcal osteomyelitis

- fungal osteomyelitis

- mucicarmine

- fungal mastoid osteomyelitis

Cryptococcosis is a disseminated fungal infection commonly affecting the lungs and the central nervous system in immunocompromised patients. Herein we report a rare case of isolated mastoid osteomyelitis due to Cryptococcus neoformans which was initially not recognized due to its uncommon presentation akin to a Betzold's abscess. A 61-year-old male presented with complaints of left ear discharge for a 1-month duration associated with a swelling in the left side of the neck for which he underwent incision and drainage without any significant improvement. a contrast-enhanced computed tomography (CT) scan revealed extensive erosion of the left mastoid cavity with a collection. Intraoperatively, red friable granulation tissue was seen within the antrum, histopathology of which revealed capsulated yeast forms. The patient underwent left canal wall down mastoidectomy along with antifungal treatment. Bone involvement is rare in patients with cryptococcal infection with vertebrae being the most common site of cryptococcal osteomyelitis. The clinical presentation is nonspecific and can pose a diagnostic dilemma, as the condition can mimic both Betzold's abscess as well as malignancy. CT findings and the use of specific fungal stains in histopathology will aid in diagnosing this condition. The purpose of this case report is to establish the first case of isolated cryptococcal mastoid osteomyelitis in the database of fungal osteomyelitis. The confirmation of fungal osteomyelitis should be based on histopathological examination. The possibility of fungal osteomyelitis should be borne in mind in any case of insidiously increasing mass with unclear etiology so that prompt antifungal therapy with surgical debridement is initiated.
\end{abstract}

\section{Introduction}

Invasive fungal infection of middle ear and mastoid is relatively rare and exclusively arises in immunocompromised patients having diabetes mellitus, prior chemotherapy, and leukemia. Cryptococcus neoformans leads to disseminated fungal infection in man and animals which is usually seen in immune-compromised patients. Cryptococcosis commonly affects the lungs and the central nervous system (CNS) with bony involvement being extremely rare. Herein we report a rare case of isolated mastoid osteomyelitis due to $C$. neoformans which was initially not recognized due to its uncommon presentation akin to a Bezold's abscess. The patient later underwent canal wall down mastoidectomy with tympanoplasty followed by antifungal treatment when intraoperative histopathology confirmed cryptococcosis.

\section{Case Report}

A 61-year-old male patient presented to the Otorhinolaryngology department with complaints of left ear discharge for a 1-month duration associated with a swelling 
in the left side of the neck for 20 days. The discharge was of insidious onset, gradually progressive, purulent, profuse, nonfoul smelling, and not bloodstained. There was no history of hard of hearing, vomiting, giddiness, tinnitus, or fever. He is a known patient of type 2 diabetes mellitus on oral hypoglycemic drugs which was well controlled. The patient was initially diagnosed to have a neck abscess at a nearby hospital and was treated with incision and drainage and antibiotics with no improvement and was referred to our institute.

On arrival, the patient was conscious and oriented. He had stable vitals with a pulse rate of $80 / \mathrm{min}$, blood pressure of $120 / 80 \mathrm{~mm} \mathrm{Hg}$, and a random blood sugar value of $161 \mathrm{mg} /$ dL. On local examination, a $2 \times 1-\mathrm{cm}$ incised wound on the left side of the neck approximately $4 \mathrm{~cm}$ below the mastoid tip over the anterior border of sternocleidomastoid was seen. There was no active pus discharge at the time of admission to the hospital. On otoscopic examination, he had a moderate central perforation in pars tensa of the left tympanic membrane. Bilateral facial nerves were intact. The hearing was near normal in both ears. Oral cavity, oropharynx, and posterior pharyngeal wall were unremarkable. There was no evidence of odontogenic or rhinogenic sources of infection.

Ultrasound evaluation of neck showed $4.7 \times 1.3 \mathrm{~cm}$ ill-defined, hypoechoic collection in the intramuscular plane. He was empirically started on intravenous crystalline penicillin, ciprofloxacin, and metronidazole. A contrast-enhanced computed tomography (CT) scan revealed extensive erosion of left mastoid cavity with thinning of sinus and dural plate and ill-defined collection tracking from the tip of mastoid along sternocleidomastoid communicating to the suboccipital region. Middle ear ossicles and inner ear structures were normal ( - Fig. 1A, B).

His total leucocyte count was $14,570 / \mathrm{mm}^{3}$ and CD4 counts, renal function tests were normal. The patient was planned for left mastoid exploration with incision and drainage of neck abscess under general anesthesia with a working diagnosis of left chronic suppurative otitis media with atticoantral disease with Bezold's abscess. The patient underwent left canal wall down mastoidectomy + type 3 tympanoplasty + conchomeatoplasty + incision and drainage of neck abscess.

On exploration, red friable granulation tissue was seen within the antrum with extensive bony erosion involving sinus plate and dural plate. Granulation tissue was noted extending into the retrosigmoid area and the vertical segment of the facial canal.

Intraoperative frozen section was sent which revealed foamy histiocytes admixed with lymphocytes and plasma cells with some suspicious forms of yeast forms of fungus and there was no evidence of malignancy. The tissue bits were sent for histopathological examination, fungal potassium hydroxide $(\mathrm{KOH})$ mount, fungal culture, and acid-fast staining.

Acid-fast staining was negative. The postoperative period was uneventful. Bilateral facial nerves were intact. Cleaning and dressing of the neck wound were done twice daily. Post aural sutures were removed on postoperative day 7 . Histopathological examination revealed multiple capsulated yeast forms that stained with Gomori Methenamine-Silver as well as with mucicarmine, suggestive of cryptococcal osteomyelitis (-Fig. 2A-C).

The patient was started on injectable fluconazole $400 \mathrm{mg} / \mathrm{d}$ for 2 weeks. An alternate day mastoid cavity pack changed and was removed on the ninth postoperative day. At the time of discharge, the patient improved symptomatically. Neck wound and the mastoid cavity were healthy. The patient was discharged on oral fluconazole $400 \mathrm{mg}$ once daily for 3 months and on fluconazole, ear drops in the left ear. On postoperative follow-up, the mastoid cavity was healthy without any evidence of persistent disease both clinically and radiologically ( - Fig. $\mathbf{3}$ ).

\section{Discussion}

Aspergillus is the most common organism causing fungal osteomyelitis. C. neoformans is a budding yeast that is ubiquitous and found mostly in areas with tall buildings where
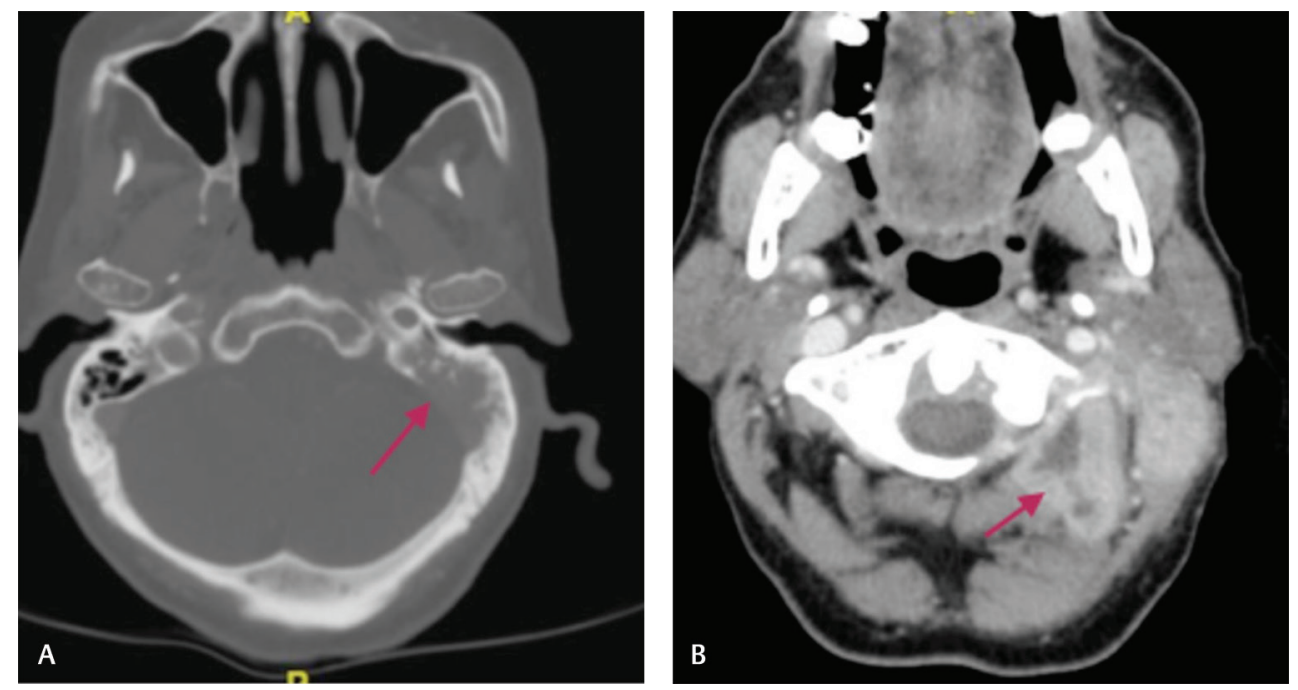

Fig. 1 A computed tomography brain showing (A) erosion of left mastoid cavity with thinning of sinus plate (marked by arrow). (B) Collection tracking from tip of mastoid in the intramuscular plane communicating with suboccipital collection (marked by arrow). 

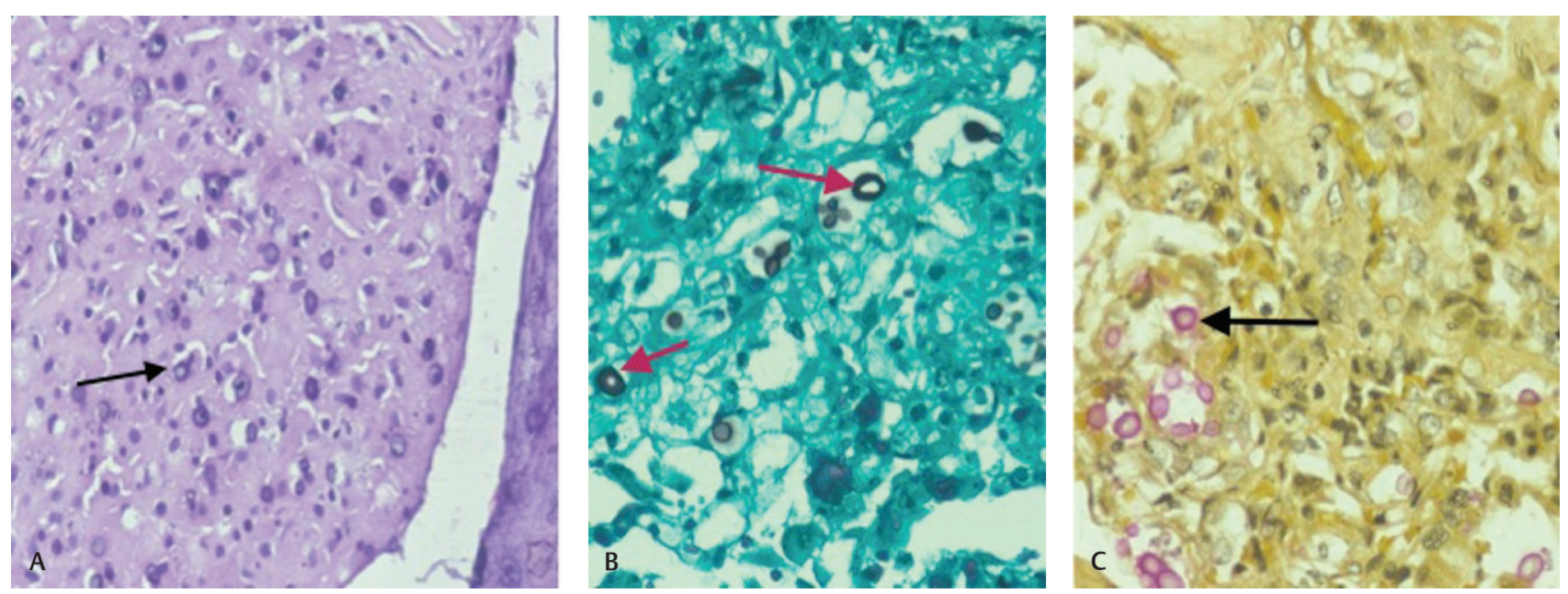

Fig. 2 Histopathological examination showing multiple capsulated yeast forms stained with (A) Hematoxylin \& Eosin 400× (B) Gomori Methenamine-Silver, and (C) mucicarmine (marked by arrows).

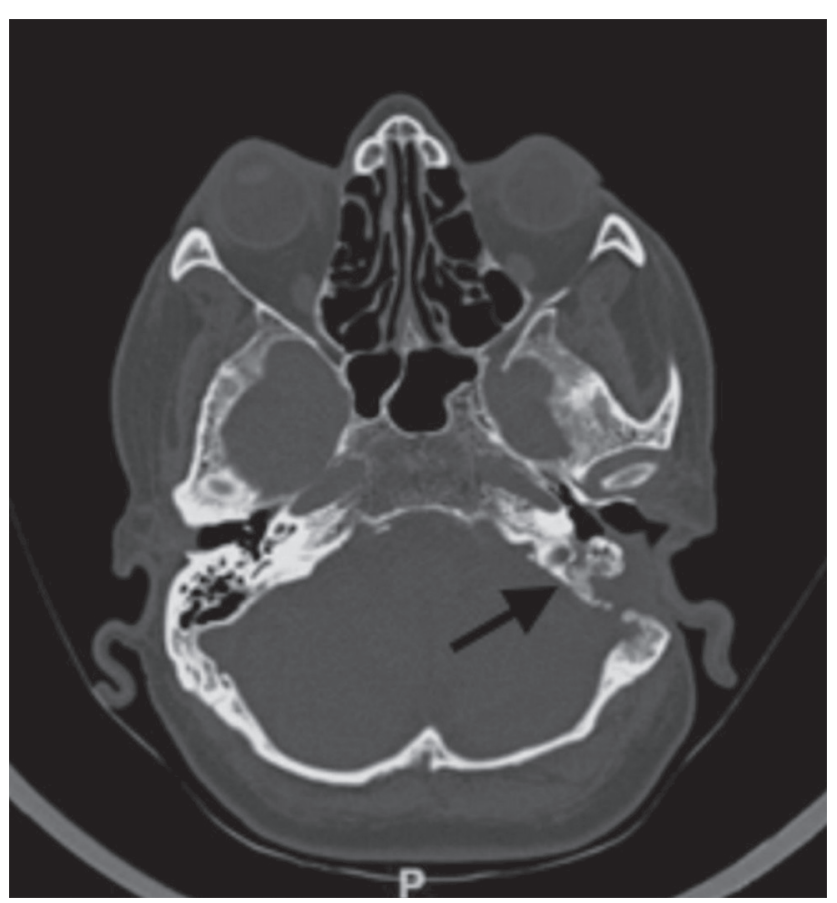

Fig. 3 CT scan showing no residual disease in left mastoid (arrow). $\mathrm{CT}$, computed tomography.

pigeons reside. It is transmitted by inhalation of conidia in bird droppings followed by a hematogenous spread in immunocompromised patients. The infection most commonly involves the lungs and the CNS while bony involvement occurs only in 5 to $10 \%$ of patients with disseminated cryptococcosis. ${ }^{1}$ Isolated osteomyelitis due to $C$. neoformans is a rare complication of disseminated cryptococcosis and around 86 cases of isolated osteomyelitis of bones have been reported in the literature based on our extensive MEDLINE search. In $75 \%$ of the cases, osteomyelitis involves only a single site with vertebrae being the most common. ${ }^{2}$ Invasive fungal infection is more often from a rhinogenic source than otologic. ${ }^{3}$ In our patient, mastoid was the only site of involvement by the fungus which is an extremely uncommon finding as per our
MEDLINE search and the most striking feature was that of an isolated otologic source. The inciting risk factor in our case is a depressed T-cell immunity caused by diabetes. The infection can extend through the soft tissue and vascular planes into adjacent structures. Without intervention, the disease is usually fatal.

Patients with cryptococcal osteomyelitis typically present with swelling and pain of the soft tissue surrounding the bone. Erythema or sinus formations are unusual. Our patient was a 61-year-old male who presented with swelling and erythema of the mastoid region along with abscess formation along sternocleidomastoid, which posed a dilemma in the diagnosis due to its presentation akin to a bacterial abscess.

The diagnosis of osteomyelitis is based on clinical, radiological, microbiological, and histological findings. Radiological examination with contrast-enhanced CT reveals a lytic lesion eroding the inner and outer tables without surrounding sclerosis with or without a bony sequestrum and overlying soft tissue mass. It also aids in ruling out a neoplasm that presents with a similar clinical picture. The absence of the above features in CT does not rule out osteomyelitis as it has poor soft-tissue resolution and fails to show marrow edema at early stages for which magnetic resonance imaging is ideal. ${ }^{4}$ Bone scan demonstrates high uptake of the isotope in the involved mastoid due to the osteoblastic reaction secondary to osteomyelitis. ${ }^{5}$ In our case, there was only mastoid erosion with a collection extending to the suboccipital region which is atypical adding up to the diagnostic dilemma. A bone scan was not done in our case.

Microbiological identification of the capsulated fungus includes India ink preparation, Giemsa staining, Meyer's mucicarmine staining, and Periodic Acid Schiff staining. Cultures are grown on Sabouraud's dextrose agar and Birdseed agar. The growth of brown-pigmented colonies is characteristic of $C$. neoformans and is due to melanin production by the organism. Biochemical confirmation of the organism is by a positive urease test, positive inositol assimilation test, and growth on 1-canavanine glycine bromothymol blue agar. In our case, Gomori methenamine silver and 
mucicarmine staining showed positivity which was further confirmed by histopathology and culture.

A cerebrospinal fluid (CSF) evaluation has proceeded in cases of doubtful CNS involvement. Cryptococcal antigen testing which detects the cryptococcal polysaccharide in the CSF is highly specific and sensitive. ${ }^{6}$ This was not done in our patient as he did not have any CNS manifestation. The CALAS test (Cryptococcal Antigen latex Agglutination System) can be used to detect the capsular polysaccharide antigens of C. neoformans in serum. ${ }^{7}$

Immunological evaluation is needed in all patients of cryptococcal infections to rule out immunosuppression. Total leucocyte counts, CD4 counts, and serology for human immunodeficiency virus (HIV) are part of immune system evaluation which were normal in our patient.

The treatment of fungal mastoid osteomyelitis includes control of underlying immunodeficient state if present, surgical debridement of all necrotic tissues along with antifungal chemotherapy. Single site cryptococcal osteomyelitis can be completely cured with fluconazole at a dose of $200 \mathrm{mg} / \mathrm{d}$ administered for a period of 10 weeks. ${ }^{8}$ The Infectious Diseases Society of America 2010 guidelines, recommend the use of $400 \mathrm{mg} / \mathrm{d}$ oral fluconazole $(6 \mathrm{mg} / \mathrm{kg}$ ) administered over a period of 6 to 12 months for nonmeningeal, nonpulmonary cryptococcosis limited to a single site. ${ }^{9}$ In our case, the patient was completely cured through injecting fluconazole for 2 weeks followed by oral fluconazole $400 \mathrm{mg}$ per day for 3 months.

\section{Conclusion}

The possibility of fungal osteomyelitis should be borne in mind as one of the differentials in any case of insidiously increasing mass with unclear etiology and unresponsive to antibiotic therapy. The purpose of this case report is to establish the first case of isolated cryptococcal mastoid osteomyelitis in an immunocompromised individual in the database of fungal osteomyelitis. The confirmation of fungal osteomyelitis should be based on histopathological examination of the specimen apart from differential fungal staining.
We further conclude that surgeons should be aware of this atypical clinical presentation of fungal osteomyelitis to ensure prompt aggressive surgical debridement along with antifungal treatment for this otherwise fatal infection.

\section{Conflict of Interest}

None declared.

\section{Acknowledgment}

I would like to express my heartfelt gratitude to Dr. Kalaiarasi for contribution in analyzing the critical points in the manuscript.

\section{References}

1 Sreenath K, Sanjeeb S, Gojendra TS, Gojen KS, Ranjana KD. Localised cryptococcal scalp osteomyelitis in an immunocompetent man-a rare case. IJARS 2014;3(4):10-12

2 Liu PYF. Cryptococcal osteomyelitis: case report and review. Diagn Microbiol Infect Dis 1998;30(1):33-35

3 Aycicek A, Kenar F, Demirdal T, et al. Facial paralysis due to invasive Aspergillus of the temporal bone in an immunocompetent child. Int J Pediatr Otorhinolaryngol Extra 2009;4:143-146

4 Raftopoulos I, Meller JL, Harris V, Reyes HM. Cryptococcal rib osteomyelitis in a pediatric patient. J Pediatr Surg 1998;33(5):771-773

5 Lee YJ, Sadigh S, Mankad K, Kapse N, Rajeswaran G. The imaging of osteomyelitis. Quant Imaging Med Surg 2016;6(2):184-198

6 Abassi M, Boulware DR, Rhein J. Cryptococcal meningitis: diagnosis and management update. Curr Trop Med Rep 2015;2(2):90-99

7 Jaye DL, Waites KB, Parker B, Bragg SL, Moser SA. Comparison of two rapid latex agglutination tests for detection of cryptococcal capsular polysaccharide. Am J Clin Pathol 1998;109(5): 634-641

8 Murphy SN, Parnell N. Fluconazole treatment of cryptococcal rib osteomyelitis in an HIV-negative man. A case report and review of the literature. J Infect 2005;51(5):e309-e311

9 Perfect JR, Dismukes WE, Dromer F, et al. Clinical practice guidelines for the management of cryptococcal disease: 2010 update by the Infectious Diseases Society of America. Clin Infect Dis 2010;50(3):291-322 